\title{
Estructuras de colaboración entre las bibliotecas nacionales de la Comunidad Europea a través de sus portales web y de las redes sociales
}

The collaboration structures among European Union national libraries in their Web portals and social networks

\author{
Carlos G. Figuerola, José Luis Alonso Berrocal, Ángel F. Zazo Rodríguez \\ Instituto Universitario de Estudios en Ciencia y Tecnología, Universidad de Salamanca, España \\ \{berrocal||figue|zazo\}@usal.es
}

\begin{abstract}
Resumen
Las bibliotecas nacionales, en cuanto que cabeceras de sus respectivos sistemas bibliotecarios, realizan diversas funciones que se ven reflejadas en sus respectivos portales web. Igualmente muchas bibliotecas nacionales han adoptado sistemas de intercomunicación específicos de la Web 2. 0, incorporándose a las redes sociales. Los hiperenlaces de estos portales hacia otros sitios web reflejan algún tipo de relación, y lo mismo sucede con seguimientos, suscripciones y flujos de mensajes a través de las redes sociales, de manera que un análisis de estos elementos podría ayudarnos a descubrir estructuras y áreas de colaboración entre ellas. En este trabajo se han analizado los portales web de las bibliotecas nacionales de los estados miembros de la Unión Europea, así como su actividad en Twitter. Se aprecia un bajo nivel de interrelación entre ellas, aunque se detecta un núcleo de bibliotecas nacionales con unos flujos de colaboración estables y un conjunto de bibliotecas periféricas, con muy débil relación con dicho núcleo.
\end{abstract}

Palabras clave: Cibermetría. Redes sociales. Twitter. Enlaces web. Bibliotecas nacionales. Europa.

\section{Introducción}

El web se ha convertido en un medio de comunicación importante; organizaciones de todo tipo lo utilizan. Como las páginas web tienen enlaces a páginas web de otras organizaciones, esos enlaces pueden reflejar al menos una parte importante de las relaciones que una organización establece con otra. Las bibliotecas nacionales de los distintos países, en cuanto que cabeceras de sus respectivos sistemas bibliotecarios, realizan diversas funciones que se ven reflejadas en sus respectivos portales web. En este sentido, los hiperenlaces de estos portales hacia otros sitios web reflejan algún tipo de relación, de manera que un análisis de tales hiperenlaces podría ayudarnos a descubrir redes y áreas de colaboración entre ellas.

\begin{abstract}
National Libraries, as their library systems' heads, perform several tasks which are depicted, to some extent, through their websites. Besides, a major group of theses national libraries also use interlinking systems from the Web 2. 0, taking part in social networks. Web links targeting other websites show some kind of relationship, and this also occurs in the social networks with followings, subscriptions and similar. In this way, studying these elements should help us to discover structures and areas of cooperation among them. In this paper, European Union national libraries' webpages are analyzed, as well as their activity in Twitter. In general, a low level of interaction between them can be appreciated. However, a central core of national libraries can be depicted, with stable relationships and collaborative networks among them. Also, a group of outlier libraries can be clearly seen, with very weak interaction with that core.
\end{abstract}

Keywords: Cybermetrics. Social networks. Twitter. Weblinks. National Libraries. Europe.

Más recientemente, muchas bibliotecas nacionales han adoptado sistemas de intercomunicación específicos de la Web 2. 0, incorporándose a las redes sociales. La posibilidad de analizar las relaciones entre las distintas bibliotecas nacionales a través de algunas de esas redes sociales también puede ayudarnos a descubrir y comprender estructuras y redes de colaboración, tal vez más informales pero no por ello menos ciertas.

Ambos aspectos, enlaces web y redes sociales tienen dinámicas diferentes y cabe preguntarse si van a mostrarnos la misma imagen de esas redes de colaboración. El propósito de este trabajo es analizar los flujos de colaboración desde ambos puntos de vista y comprobar si ambos ámbitos (web y redes sociales) tienen comportamientos parecidos o no. 
Este trabajo está organizado como sigue: en el capítulo siguiente se hace una breve introducción al estado del arte en este campo, para, a continuación, exponer los principales principios metodológicos seguidos en nuestra investigación. Después se expondrán los principales resultados obtenidos del análisis cibermétrico y, a continuación, los resultados obtenidos con el análisis de redes sociales. Finalmente, se ofrecen unas conclusiones.

\section{Trabajos previos}

Los estudios sobre la estructura de los hiperenlaces entre las páginas web se realizan desde hace ya bastantes años. Tal vez uno de los primeros fue el de Almind e Ingwersen (1997), acuñando, de paso, el término Webmetría. El mismo Ingwersen (1998) introdujo el factor de Impacto Web (WIF) que, aunque hoy ya no se utiliza, supuso un hito importante en este campo del conocimiento. Thelwall (2003) y Bar-llan $(2004,2005)$ estudiaron la naturaleza de los enlaces web. Una cantidad importante de análisis cibermétricos sobre aspectos o sitios concretos se han realizado, pero tal vez son especialmente abundantes sobre sitios web de corte académico como universidades e instituciones de educación superior. En este sentido, si nos centramos en Europa, Thelwall, Tang y Price (2003) estudiaron los webs de universidades de 16 países europeos y encontraron que la mitad de todas las páginas estudiadas estaban en inglés, y tenían, además, un fuerte nivel en interenlazado. El otro gran bloque de hiperenlaces se producía entre páginas que estaban en la misma lengua.

Heimeriks y Van der Besselaar (2006) analizaron los webs académicos de los 15 países que en 2006 formaban la Unión Europea encontraron que el hiperenlazado seguía patrones linguísticos y también de tipo geográfico (mismo país, cercanía geográfica, etc.). Estos patrones eran menos marcados cuando los datos se estudiaban a nivel de departamentos universitarios.

Ortega y Aguillo (2008a) y Ortega y otros (2008d) recopilaron datos de 530 universidades europeas en 2005; y trazaron un mapa de hiperenlaces entre los sistemas universitarios de los distintos países. Thelwall y Zuccala (2008) trazan también un mapa de hiperenlaces de sistemas nacionales de educación superior, buscando hallar posibles efectos de las ampliaciones de la Comunidad Europea (2004 y 2007).

Las universidades del Reino Unido han sido objeto de varios estudios webométricos, en buena medida propiciados por la disponibilidad de datos a través del Academic Web Link Database Project (http://www.scit.wlv.ac. uk/ cm1993/cybermetrics/ database/), descrito por M. Thelwall (2001). En Thelwall (2002) y Thelwall y Harries (2003), utilizando datos de 108 universidades del Reino Unido, se aplicaron diferentes modelos de documento alternativos, superando la equiparación mecánica entre documento y página web. Björneborn (2004), trabajando sobre datos de los webs de esas mismas 108 universidades del Reino Unido, estudió sus propiedades de small-world, y la aplicación del modelo bow-tie. Wilkinson y otros (2003) utilizan también datos de universidades británicas para su trabajo sobre las motivaciones de los hiperenlaces, y Payne y Thelwall (2004) utilizaron los datos correspondientes al año 2002 de 111 universidades del Reino Unido para identificar los modelos matemáticos que mejor caracterizan la estructura de enlaces del web académico británico. En Stuart, Thelwall y Harries (2007), se ocuparon de las relaciones entre el mundo académico, las instituciones gubernamentales y sitios de tipo comercial a través de sus hiperenlaces.

Por lo que se refiere a las universidades escandinavas, desde un punto de vista webométrico, contamos con el trabajo de Ortega y Aguillo (2008b); el de Aaltojärvi y otros (2008) y los de Holmberg y Thelwall (2009) y el de Holmberg (2010) sobre Finlandia, centrados en el gobierno local o municipal, siempre desde el punto de vista cibermétrico. Sobre los hiperenlaces del mundo académico alemán tenemos el trabajo de Ortega y Aguillo (2008c). El mismo Ortega había efectuado anteriormente un estudio sobre las relaciones, a partir de los enlaces web de universidades, gobierno e industria entre instituciones de investigación alemanas y españolas, dentro del ámbito biosanitario (Ortega Priego, 2003).

Sobre España, siempre dentro de la webometría, hay que mencionar los estudios de BaezaYates, Castillo y López (2005) sobre el web español en general, pero en el que parece tener un importante peso el web universitario. $Y$ también los de Ortega y Aguillo (Ortega y Aguillo, 2007a; Ortega Priego y Aguillo Caño, 2007b); éstos ya referidos específicamente al ámbito universitario.

Las redes sociales, los blogs, o cualquier foro en línea han convertido a Internet en un gran repositorio de comentarios de los que se pueden extraer múltiples informaciones (Thelwall, Buckley y Paltoglou, 2011), tal y como muestran los numerosos trabajos de investigación que se vienen realizando en los últimos años. 
De todas las redes sociales actuales nos vamos a centrar en twitter. La característica principal de este modo de comunicación reside en la longitud de sus mensajes, llamados tweets, que se muestran en la página principal del usuario. Los usuarios pueden suscribirse a los tweets de otros usuarios, lo que se denomina "siguiendo" -a los suscriptores se les llaman "seguidores"- . Por defecto, los mensajes son públicos, pudiendo difundirse de manera privada mostrándolos únicamente a seguidores. Este método se utiliza para compartir información y también para describir prácticamente cualquier actividad diaria (Java, 2009), ya que permite que los usuarios puedan expresar sus opiniones e intereses, de forma abreviada y altamente personalizada en tiempo real (García Esparza, 2012). Su importancia se muestra en que está presente en prácticamente todas las áreas de la vida (social, económica, educativa...) y abarca cualquier tema (deportes, cultura, ocio, industria, ciencia...).

Si valoramos la importancia de esta red social en términos cuantitativos, es necesario hacer referencia al volumen de tweets que se generan a diario -500 millones que se han duplicado en el último año-. Si la valoración la hacemos en términos cualitativos, es necesario considerar cómo su influencia se refleja en la multitud de actos sociales que se retrasmiten en tiempo real con el objetivo de ganar en visibilidad, así como en el gran número de mensajes no originales que se vuelven a difundir (retweets); por lo que se puede considerar incluso que Twitter llega a constituir nichos de opinión, ya que un mensaje creado por una persona (ya sea original o un fragmento de otra obra como un titular de un periódico o un extracto de una noticia) puede ser retwitteado por otro $u$ otros usuarios que, a su vez, lo vuelven a retransmitir, provocando un efecto de difusión en forma de racimo.

Es cierto que gran parte de la información que aportan los tweets es completamente irrelevante, además es necesario considerar que en muchos casos los mensajes aislados de su contexto pierden valor, pero también es una fuente de información muy rica, porque recoge de manera condensada la información relevante para los usuarios, ya sean personas individuales, instituciones o empresas, que son los que destacan noticias, opiniones o sentimientos, información que resultaría muy difícil de recopilar por otros canales. Y, precisamente por esto, el análisis de los mensajes de twitter está siendo utilizado como materia de base para múltiples investigaciones que van desde el papel que juegan los distintos tipos de usuarios en la difusión de la información (Cha, 2012) a análisis sociológicos (Chen, 2012), aplicaciones a la clasificación (Lee, 2011) y recuperación de la información (Garcia Esparza, 2012; Yerva, 2012), análisis semántico (Narr, 2011) o aplicados a sectores concretos como el campo semántico de los libros electrónicos (Alonso Berrocal et al., 2012).

Otro aspecto fundamental de estudio en estos momentos es analizar la influencia real, la credibilidad de las redes sociales (Westerman, 2012; Metzger, 2010). En la actualidad hay dos algoritmos que tratan de medir esta influencia, Klout y Peerlndex.

Klout (http://www.klout.com) es la herramienta que mayor aceptación está teniendo en estos momentos; y para muchos es un estándar de influencia. Mide la influencia de los perfiles, en un rango entre 0 y 100 y trabaja con unos 30 algoritmos, no conocidos. La medición se realiza (Serrano-Puche, 2012) teniendo en cuenta el número de personas sobre las que se ejerce la influencia; el control que se hace mediante el retweet de la información; y la influencia que también tienen los seguidores del perfil en cuestión. Sobre el trabajo con Klout disponemos de varios trabajos interesantes (Berry, 2010; Edwards, 2013).

Por otro lado Peerlndex (http://www. peerindex. $\mathrm{com} /$ ) intenta diferenciarse valorando la noción de autoridad y reputación. Para ello se controlan (Serrano-Puche, 2012) el modo en el que los demás perfiles confían en tus opiniones, midiendo el impacto de lo que hacemos en nuestros seguidores, y la frecuencia en la que actualizamos nuestro estatus con respecto a los demás.

\section{Metodología}

Para este trabajo se han tomado los sitios web de las bibliotecas nacionales de los estados miembros de la Comunidad Europea. El concepto de biblioteca nacional no tiene exactamente el mismo significado en todos los países; así, en Italia podemos encontrar, según las fuentes empleadas, dos o tres bibliotecas nacionales, como se aprecia en Wikipedia, aun cuando esto es claramente una excepción. Por lo que se refiere a análisis de sitios y enlaces web, hemos localizado el portal web de cada una de las bibliotecas nacionales y también, según los casos, se ha identificado el dominio propio de cada una de ellas.

En este sentido, hay que decir que todas tienen dominio web propio, aunque no siempre de primer nivel; $y$ en bastantes de ellas el sitio principal se ve acompañado por otros sitios web 
que lo complementan. Es el caso, por citar un ejemplo, de la Biblioteca Nacional de Francia, con su sitio principal (http://www.bnf.fr) y satélites como Gallica (http://gallica.bnf.fr, su biblioteca digital); en sentido distinto, en España su Biblioteca Nacional tiene integrado en el mismo sitio la biblioteca digital, mientras que la hemeroteca digital tiene su propio portal web (http://hemerotecadigital.bne.es), aunque dentro del mismo dominio.

Para todas ellas hemos recogido datos procedentes de dos fuentes distintas: algunos datos globales extraídos de Alexa (http://alexa.com) y, de otro lado, datos obtenidos a partir de la utilización de un crawler. En el primer caso, Alexa es un servicio comercial de análisis de tráfico web que ofrece de forma libre alguna información acerca de cualquier portal o dominio; la información que ofrece en abierto, sin embargo, el propio Alexa la califica de no certificada, por lo que conviene tomarla con cierta precaución.

Respecto al uso de crawlers para la obtención de datos sobre enlaces, se trata de una opción obvia si se tienen en cuenta las irregularidades y restricciones en los servicios ofrecidos por los buscadores al uso. En el pasado tales servicios han sido la fuente básica de numerosos análisis cibermétricos, pero muchos de tales servicios ya no están disponibles. Así pues, hemos optado por el uso de un par de crawlers. Básicamente hemos utilizado nuestro propio crawler (Alonso Berrocal y otros, 2006), completando o contrastando datos cuando ha sido preciso con la aplicación del conocido wget (http://www.gnu.org/ software/wget). Esto nos ha permitido descargar todas las páginas, hasta un nivel determinado de recursión (en nuestro caso, siete) de los sitios o dominios web de cada Biblioteca Nacional, y obtener todos los enlaces existentes en dichas páginas. Estos datos han sido el núcleo central de nuestro análisis cibermétrico.

El trabajo con twitter se ha llevado a cabo analizando los perfiles de la Tabla I mediante la API que suministra la red social. Para el estudio de la credibilidad de la red social se ha trabajado con la API de Klout. Para ello se ha creado un crawler según las especificaciones de Alonso Berrocal et al. (2012), añadiendo la posibilidad de recoger la información suministrada por Klout. Se decidió no recoger la información con Peerlndex debido a que no todas las bibliotecas tenían información recogida con este índice en el momento de la recogida de datos.

En este trabajo de twitter nos interesa sobre todo el análisis de las menciones existentes y que caracterizan más los tweets analizados en los perfiles que estamos siguiendo, lo que de- termina las cohesiones existentes en las entidades de usuario analizadas.

\begin{tabular}{ll}
\hline Perfil Twitter & País \\
\hline DNB_Aktuelles & Alemania \\
\hline matica_sk & República Eslovaca \\
\hline knjiznicaNUK & Eslovenia \\
\hline BNE_biblioteca & España \\
\hline NationalLibrary & Estonia \\
\hline ActuBnF & Francia \\
\hline nemzetikonyvtar & Hungría \\
\hline NLIreland & Irlanda \\
\hline LNB_Iv & Letonia \\
\hline KB_Nederland & Holanda \\
\hline PWr_Wroclaw & Polonia \\
\hline britishlibrary & Reino Unido \\
\hline narodniknihovna & República Checa \\
\hline kungbib & Suecia \\
\hline
\end{tabular}

Tabla I. Perfiles analizados

La retransmisión de tweets por parte de un perfil - es decir, la difusión de la información considerada más interesante para ese perfil- permite diferentes estudios, entre ellos determinar los aspectos más interesantes que la comunidad considera dignos de ser difundidos. Para analizar la influencia de los tweets de un determinado perfil, se calculan los retweets que se han realizado, poniendo en relación, la ratio tweet/retweet y el número total de retweets que ha tenido frente al número de tweets que tiene el perfil. Esto determinará la importancia de los mensajes de los perfiles en función de la importancia que se le da por parte de otros perfiles.

En el caso de Klout obtenemos la información del score asignado para cada una de las cuentas, determinando la influencia asignada por los algoritmos. También obtenemos los 5 perfiles a los que más se influye y los 5 que más nos influyen. Obtenemos asimismo los 5 topics más relevantes de cada uno de los perfiles.

\section{Resultados}

\subsection{Análisis cibermétrico}

Los sitios web de las bibliotecas nacionales arrojan un promedio de páginas recogidas de 
1.495, con una media también de 144.378 enlaces internos a cada dominio y 6.609 enlaces exteriores, hacia otros dominios.

\begin{tabular}{|c|c|c|c|c|}
\hline Biblioteca & $\begin{array}{c}\text { Inlinks } \\
\text { Alexa }\end{array}$ & Págs. & $\begin{array}{r}\text { Links } \\
\text { internos }\end{array}$ & $\begin{array}{r}\text { Links } \\
\text { externos }\end{array}$ \\
\hline Alemania & 453 & 334 & 13250 & 1194 \\
\hline Austria & 2308 & 4272 & 137962 & 4518 \\
\hline Bélgica & 1086 & 377 & 18140 & 953 \\
\hline Bulgaria & 310 & 1647 & 170154 & 11773 \\
\hline Chequia & 1231 & 5856 & 1297895 & 56612 \\
\hline Chipre & 48 & 531 & 4056 & 3735 \\
\hline Dinamarca & 2063 & 14392 & 1363282 & 7404 \\
\hline Eslovaquia & 196 & 259 & 44003 & 8313 \\
\hline Eslovenia & 6263 & 298 & 13623 & 2058 \\
\hline España & 4575 & 1130 & 38854 & 4895 \\
\hline Estonia & 752 & 2102 & 429503 & 27483 \\
\hline Finlandia & 198 & 521 & 13491 & 8900 \\
\hline Francia & 14023 & 983 & 155227 & 3871 \\
\hline Grecia & 694 & 80 & 6352 & 30 \\
\hline Holanda & 3067 & 1308 & 47094 & 5656 \\
\hline Hungría & 3259 & 641 & 53583 & 7056 \\
\hline Irlanda & 1405 & 1690 & 48059,4 & 222671 \\
\hline Italia (Nápoles) & 220 & 478 & 14891 & 58,24 \\
\hline Italia (Roma) & 9729 & 115 & 4090 & 605 \\
\hline Italia (Florencia) & 2336 & 298 & 6288 & 1792 \\
\hline Letonia & 398 & 405 & 21367 & 7914 \\
\hline Lituania & 424 & 180 & 13109 & 631 \\
\hline Luxemburgo & 3767 & 143 & 5864 & 322 \\
\hline Malta & 37 & 37 & 1272 & 140 \\
\hline Polonia & 1198 & 961 & 15028 & 1478 \\
\hline Portugal & 629 & 1602 & 51526 & 5874 \\
\hline Reino Unido & 15232 & 816 & 21595 & 1228 \\
\hline Rumania & 393 & 842 & 19437 & 3784 \\
\hline Suecia & 2465 & 1279 & 61662 & 3933 \\
\hline
\end{tabular}

Tabla II. Datos de enlaces y páginas

Aunque en los sitios web actuales, constituídos en su mayoría por aplicaciones web que generan páginas dinámicas, el número de éstas tiene poca relevancia, sí es destacable el elevado número de enlaces por página, 100,93 de media $\mathrm{y}$, particularmente notable, la abultada diferencia entre enlaces internos, hacia páginas del propio sitio o dominio web, y externos, hacia otros sitios web.

La Tabla II muestra la cantidad de páginas recogida para cada biblioteca nacional, al igual que los links internos y externos recogidos por nuestro crawler; también muestra la cantidad de inlinks recibidos según Alexa.com.

Un mero análisis de correlación entre estas series de datos muestra la desconexión entre los datos de Alexa y los obtenidos con el crawler, pero también la disociación entre los propios datos procedentes de Alexa (inlinks y ranking mundial de tráfico), lo que hace dudar de esta fuente.

Por lo que se refiere a los datos obtenidos por el crawler, el coeficiente de Pearson muestra fuerte correlación entre el número de páginas y la cantidad de enlaces internos (0.89), lo cual era de esperar; pero, sin embargo, muestra disociación entre número de páginas y enlaces hacia el exterior (Pearson=0.11) y también entre la cantidad de enlaces internos y externos (Pearson= 0.14).

Si nos centramos en las relaciones web entre unas bibliotecas nacionales y otras, lo primero que hay que hacer notar es el notablemente bajo nivel de interconexión entre ellas: Todas mantienen algunos enlaces entre sí, pero el número de tales enlaces es muy pequeño.

Si consideramos el grafo formado exclusivamente por las bibliotecas nacionales y sus hiperenlaces entre ellas, podemos observar los datos de in y out degree en las tablas III y IV.

\begin{tabular}{lc}
\hline Biblioteca & Grado de Salida \\
\hline Francia & 12 \\
\hline Reino Unido & 12 \\
\hline Holanda & 10 \\
\hline Estonia & 5 \\
\hline Suecia & 5 \\
\hline Austria & 4 \\
\hline Bélgica & 4 \\
\hline España & 4 \\
\hline Italia (Florencia) & 4 \\
\hline Chequia & 3 \\
\hline
\end{tabular}

Tabla IIII. Grado de salida de las bibliotecas 


\begin{tabular}{lr}
\hline País & Grado de entrada \\
\hline Italia (Nápoles) & 11 \\
\hline Portugal & 7 \\
\hline Suecia & 6 \\
\hline Bélgica & 6 \\
\hline Chequia & 5 \\
\hline Dinamarca & 5 \\
\hline Luxemburgo & 4 \\
\hline Austria & 3 \\
\hline España & 3 \\
\hline Italia (Roma) & 3 \\
\hline
\end{tabular}

Tabla IV. Grado de entrada de las bibliotecas
La Figura 1 muestra el grafo formado por las Bibliotecas nacionales y sus enlaces, distribuidas según el algoritmo Force Atlas (Medrano, Berrocal y Figuerola, 2011), tendiendo a colocar más cercanas las que tienen un mayor número de enlaces entre sí. El tamaño de los nodos, de otro lado, es proporcional al grado de cada biblioteca. Esta figura muestra claramente el bajo nivel de entrelazado entre las bibliotecas nacionales europeas. Algunas aparecen claramente como periféricas (las de Grecia, Chipre, Lituania, Letonia, Bulgaria, Polonia), pero el resto tampoco muestra gran cohesión entre ellas. El núcleo más central gira en torno a las bibliotecas nacionales británica y francesa, a las cuales acompañan las bibliotecas nacionales de España, Chequia, Holanda, Austria y, algo más lejos, Dinamarca, Suecia, Finlandia, las bibliotecas nacionales italianas, la alemana, la de Portugal, Hungría, Bégica, Estonia o Luxemburgo.

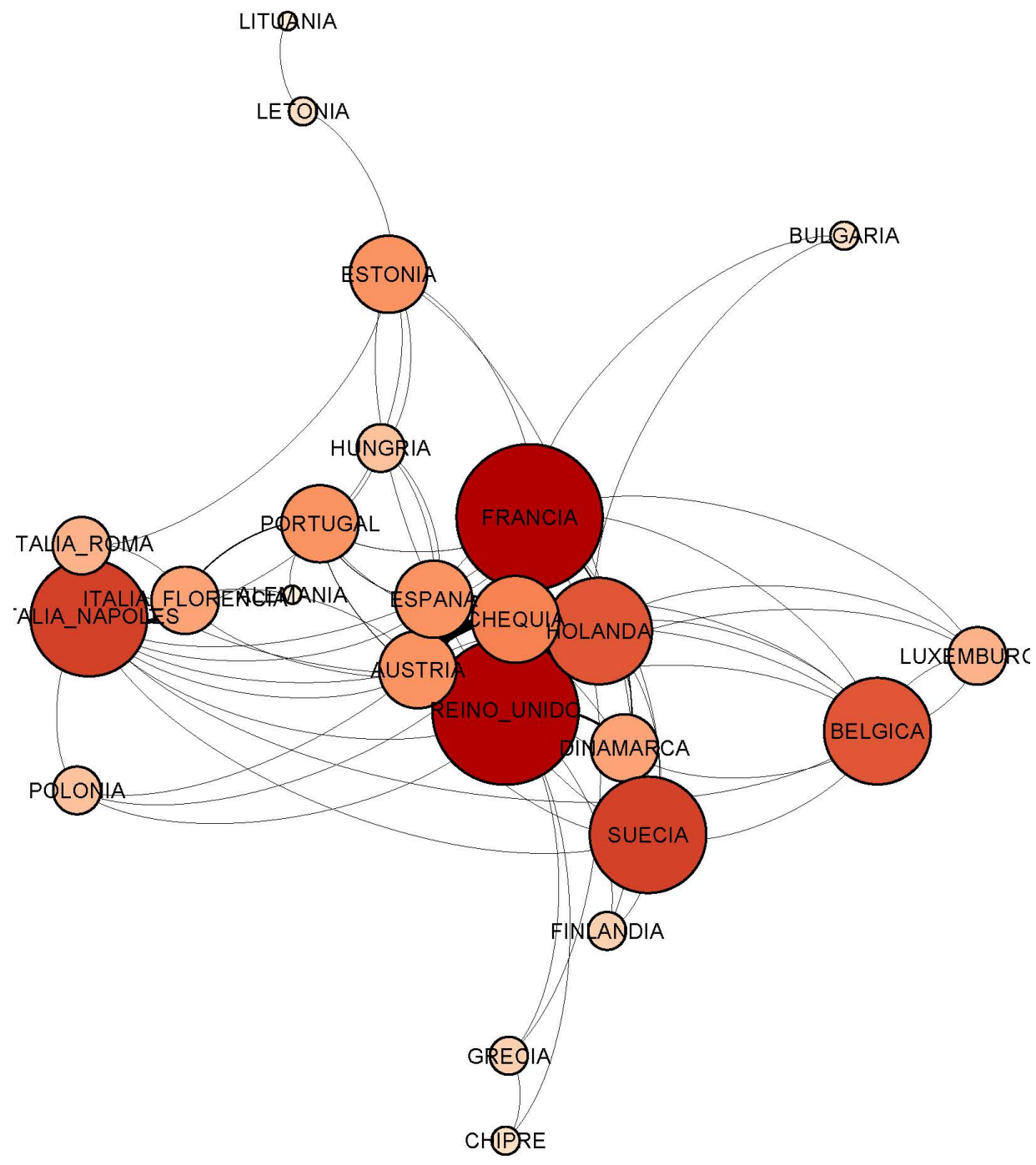

Figura 1. Grafo de las Bibliotecas Nacionales 
La Tabla $V$ (en apéndice) y la Figura 2 (en apéndice) muestran los sitios web más enlazados por las bibliotecas nacionales, junto con el número de bibliotecas nacionales que enlazan con cada uno de ellos. Destacan sitios obvios, como The European Library y Europeana, así como sitios claramente relacionados con los servicios técnicos bibliotecarios que las bibliotecas nacionales soportan (ifla.org, issn.org, etc.). Otros son enlaces a software de uso común (adobe.com, por ejemplo), y otros apuntan a redes sociales de diverso tipo, frecuentes en portales de muy diversas clases (youtube, twitter, facebook, flickr, etc).

\begin{tabular}{llllll}
\hline Sitio web & BNs & Sitio web & BNs & Sitio web & BNs \\
\hline europeanlibrary. org & 24 & ebscohost. com & 10 & itunes. apple. com & 6 \\
\hline europeana. eu & 24 & isbn-international. org & 9 & impact-project. eu & 6 \\
\hline facebook. com & 21 & ubka. uni-karlsruhe. de & 8 & ebrary. com & 6 \\
\hline loc. gov & 20 & stanford. edu & 8 & dublincore. org & 6 \\
\hline twitter. com & 17 & oclc. org & 8 & cenl. org & 6 \\
\hline ifla. org & 17 & iso. org & 8 & archive. org & 6 \\
\hline google. com & 17 & doaj. org & 8 & vimeo. com & 5 \\
\hline youtube. com & 15 & worldcat. org & 7 & viaf. org & 5 \\
\hline unesco. org & 15 & oxfordartonline. com & 7 & udcc. org & 5 \\
\hline w3. org & 13 & microsoft. com & 7 & sciencedirect. com & 5 \\
\hline issn. org & 13 & libereurope. eu & 7 & minervaeurope. org & 5 \\
\hline europa. eu & 13 & jstor. org & 7 & iaml. info & 5 \\
\hline adobe. com & 13 & getty. edu & 7 & gutenberg. org & 5 \\
\hline ismn-international. org & 12 & wdl. org & 6 & flickr. com & 5 \\
\hline wikipedia. org & 11 & slideshare. net & 6 & creativecommons. org & 5 \\
\hline emeraldinsight. com & 11 & purl. org & 6 & & \\
\hline cerl. org & 11 & oxfordreference. com & 6 & & 5 \\
\hline & 12 & a & & 5 \\
\hline
\end{tabular}

Tabla V. Webs más enlazados desde las bibliotecas nacionales

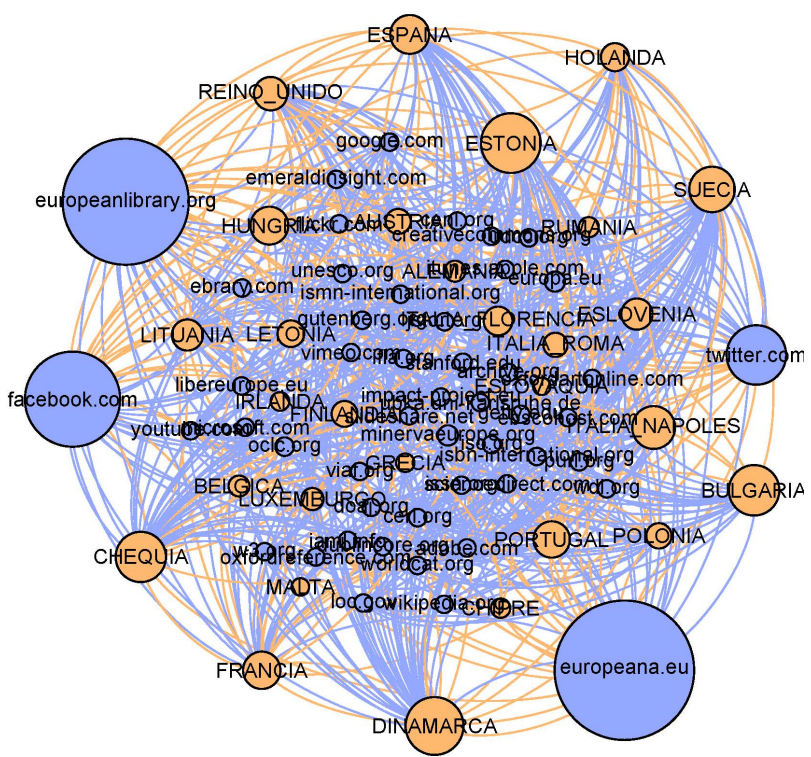

Figura 2. Grafo de webs más enlazados desde las bibliotecas nacionales 
A dublincore.org sólo apuntan seis bibliotecas, todas con unos pocos enlaces, algo que contrasta con un supuesto uso bastante extendido de este esquema de metadatos.

Por lo que se refiere a URLs persistentes, sólo una Biblioteca Nacional, la de Estonia, mantiene enlaces con handle.net, mientras que seis bibliotecas apuntan a purl.org, especialmente las de Finlandia y Suecia, con abundantes enlaces.

Al consorcio W3C sólo apuntan 13 de las Bibliotecas analizadas, a pesar de que el enlace que certifica la conformidad con el estándar HTML es habitual es sitios institucionales. Menor cobertura, aparentemente, tiene el estándar WCAG de accesibilidad web, cuyo enlace de conformidad sólo aparece en 5 de los portales analizados. Sólo 5 bibliotecas nacionales apuntan a Creative Commons, y de ellas sólo la de Dinamarca parece utilizarla abundantemente. Enlaces a licencias de software libre (GNU/GPL) sólo aparecen en dos bibliotecas nacionales, entre ellas la portuguesa, que lo aplica con profusión.

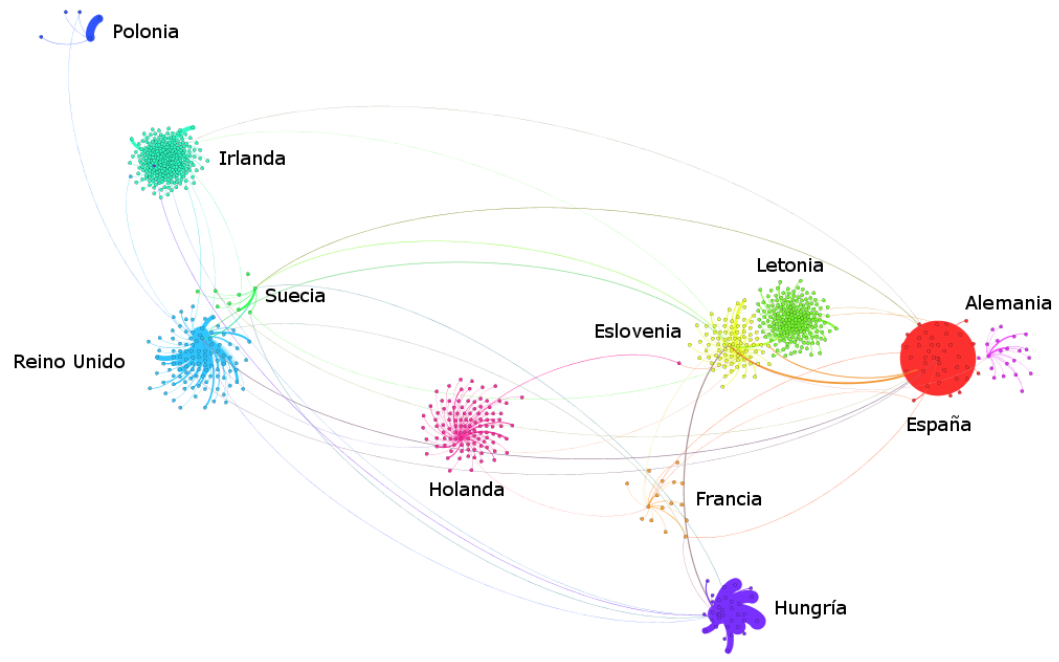

Figura 4. Grafo de menciones twitter entre los amigos

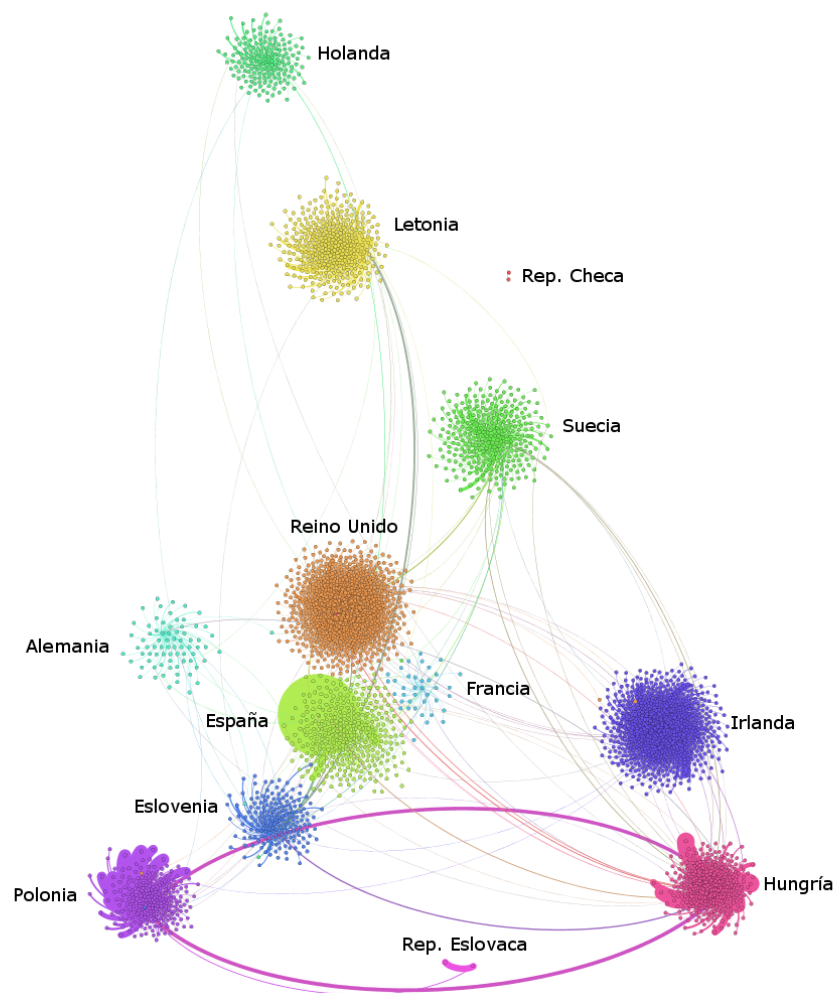

Figura 5. Grafo de menciones twitter entre todos 
Cuando analizamos el grafo obtenido de las menciones existentes en los tweets observamos que tanto el grafo que se ha realizado solamente con los amigos de los perfiles (Figura 4), así como el formado por todas las entidades (Figura 5 ) tienen una estructura muy similar en su comportamiento. Calculando el grado de intermediación de ambos grafos tenemos los datos que se reflejan en la Tabla VI.

\begin{tabular}{cc}
\hline Amigos & Todos \\
\hline Reino Unido & Reino Unido \\
\hline Irlanda & España \\
\hline Holanda & Irlanda \\
\hline España & Holanda \\
\hline
\end{tabular}

Tabla VI. Bibliotecas según su betweenness

Como podemos ver en la Tabla VI el cambio más significativo es el de España, que, teniendo en cuenta todas las menciones, se pone en segundo lugar. Esto se pone en relación con los datos obtenidos para el índice Klout.

En función del índice Klout el siguiente grafo (Figura 6) nos muestra los valores más importantes, reflejando las relaciones existentes entre los diferentes perfiles.
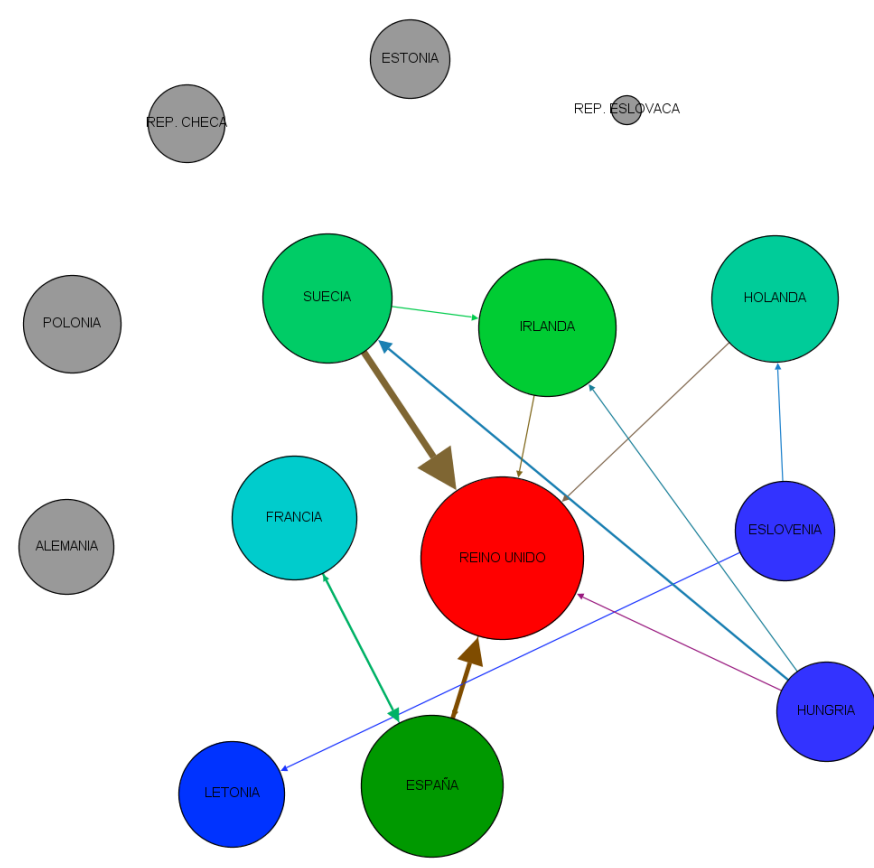

Figura 6. Grafo según klout y relaciones entre ellas

El mayor índice de influencia lo tiene Reino Unido, seguido de España, Irlanda, Suecia y
Holanda. Los enlaces nos muestran las menciones existentes a los otros perfiles y, como podemos observar se producen desde Suecia al reino Unido, y desde España al Reino Unido.

Si tenemos en cuenta la influencia de los retweets generados los datos son los siguientes:

\begin{tabular}{lcc}
\hline & tweet/retweet & retweet total/tweet \\
\hline Alemania & 0,56 & 2,72 \\
\hline República Eslovaca & 0,00 & 0,00 \\
\hline Eslovenia & 0,46 & 1,33 \\
\hline España & 0,85 & 8,48 \\
\hline Estonia & 0,02 & 0,02 \\
\hline Francia & 0,76 & 2,67 \\
\hline Hungría & 0,21 & 0,35 \\
\hline Irlanda & 0,15 & 0,71 \\
\hline Letonia & 0,39 & 1,42 \\
\hline Holanda & 0,42 & 0,92 \\
\hline Polonia & 0,33 & 2,18 \\
\hline Reino Unido & 0,87 & 12,00 \\
\hline República Checa & 0,23 & 0,33 \\
\hline Suecia & 0,41 & 1,00 \\
\hline
\end{tabular}

Tabla VII. Influencia de los retweets.

En la Tabla VII si ordenamos por el criterio tweet/retweet el orden es Reino Unido, España, Francia y Alemania; si ordenamos por el criterio retweet total/tweet el orden es Reino Unido, España, Alemania y Francia. Como podemos ver la influencia que tienen sus retweets se mantiene de forma estable.

\section{Conclusiones}

En los aspectos cibermétricos, en especial en el análisis de hiperenlaces, lo primero que hay que destacar es el bajo nivel de interenlazado en general que se da entre las bibliotecas.

Pese a ello, en el grafo formado por las bibliotecas nacionales y sus enlaces se puede apreciar claramente un núcleo y una periferia; pero incluso la cohesión de ese núcleo es pequeña y la periferia aparece casi totalmente desconectada del núcleo. Este patrón se reproduce en el análisis de twitter coincidiendo el núcleo central del análisis cibermétrico y el núcleo central del análisis de twitter.

Contrasta esta poca cohesión del grafo con la estructura de los nodos individuales que, en términos generales, muestran signos de madu- 
rez y estabilidad, como claramente indican el número medio de páginas y también el número de enlaces salientes por página. Sucede que tales enlaces son en su gran mayoría internos, a otras páginas de la misma biblioteca.

Lo enlaces salientes a otros sitios o dominios web lo son, en una gran parte, a sitios de interés técnico bibliotecario (ISSN, ISBN, IFLA, etc). Destacan, entre los sitios externos más enlazados, los de Europeana y The European Library $\mathrm{y}$, a continuación, dos de las redes sociales más importantes: Facebook y Twitter. Estos cuatro sitios web tienen un poder de intermediación notable, puesto que no sólo reciben enlaces de las bibliotecas, sino que también ellos apuntan hacia las propias bibliotecas. En el caso de Facebook y Twitter, claramente actúan como un nexo de comunicación entre las bibliotecas nacionales, complementando la actuación de los hiperenlaces de los sitios de las Bibliotecas.

En este sentido, cabría pensar que tales hiperenlaces reflejan una colaboración más formal, de corte más institucional; mientras que la intecomunicación a través de las redes sociales es desde luego mucho más informal y, si se quiere, espontánea.

En el análisis de twitter podemos observar algunas coincidencias notables, como es el caso del perfil europenaeu mayoritariamente enlazado desde la mayor parte de los perfiles. Otros como ifla, europeanlibrary o impactocr se enlazan menos, pero, curiosamente, sirven de nexo de unión entre algunos de los perfiles (Figura 6).

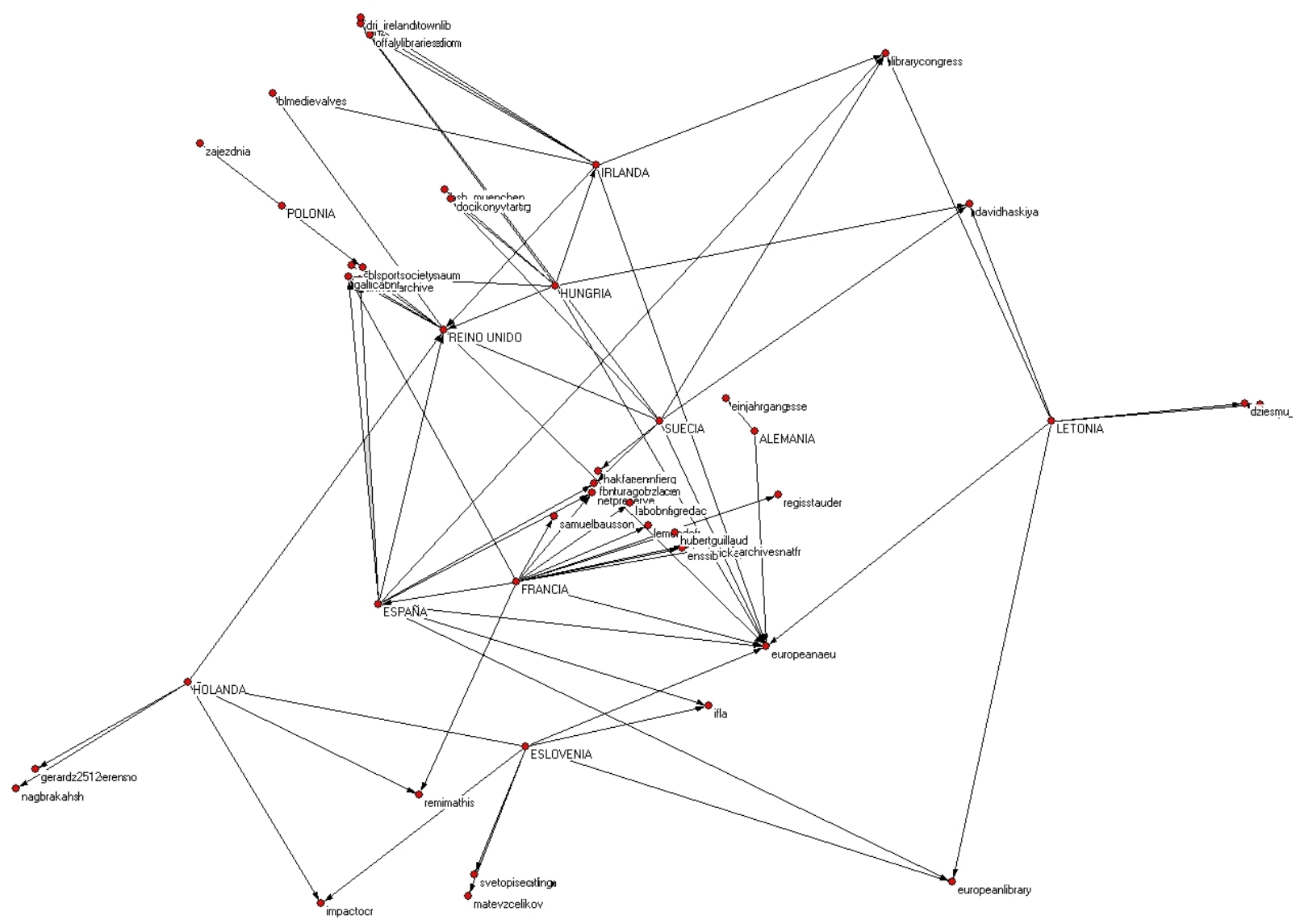

Figura 6. Grafo MDS para visualizar entidades externas

Pero twitter nos ofrece otra información, como es el índice de influencia de los perfiles, representado por el índice klout y por la influencia de los retweets, que determinan que Reino Unido y España son de las más influyentes en el conjunto de las bibliotecas nacionales.

\section{Referencias}

Aaltojärvi, I.; Arminen, I.; Auranen, O.; Pasanen, H. (2008). Scientific productivity, web visibility and citation patterns in sixteen Nordic sociology departments. // Acta Sociologica. 51:1, 5-22.

Almind, T. C.; Ingwersen, P. (1997). Informetric Analyses on the World Wide Web: Methodological Approaches to 'webometrics'. // Journal of Documentation. 53:4, 404426. 
Alonso Berrocal, José Luis; García Figuerola Paniagua, Carlos; Zazo Rodríguez, Ángel Francisco (2006). SACARINO (Sonda AutomátiCA para la Recuperación de INformación en la Web): un robot para recorrer y procesar la Web. // Scire: representación y organización del conocimiento. 12:1 (en.-jun. 2006) 211-224.

Alonso Berrocal, José Luis; Gómez Díaz, Raquel; García Figuerola Paniagua, Carlos; Zazo Rodríguez, Ángel Francisco; Cordón García, José Antonio (2012). Propuesta de estudio del campo semántico de los libros electrónicos en Twitter. // Scire: representación y organización del conocimiento. 18:2 (jul.-dic. 2012) 97-97.

Alonso Berrocal, J. L.; Figuerola, Carlos G.; José Federico Medrano (2011). Visualización de grafos Web. // Avances en Informática y Automática. Quinto Workshop. Salamanca, 2011, 167-190.

Baeza-Yates, R.; Castillo, C.; López, V. (2005). Characteristics of the Web of Spain. // Cybermetrics, 9:1.

Bar-llan,J. (2004). A microscopic link analysis of academic institutions within a country-the case of Israel. // Scientometrics. 59, 391-403.

Bar-llan, J. (2005). What do we know about links and linking? A framework for studying links in academic environments. // Information Processing \& Management. 41:4, 973-986.

Berry, M. (2010). Better Know the Klout Classes. Measuring Online Influence. The Official Klout Blog. Publicado el 2 de agosto de 2010. Url: http://corp.klout.com/blog/2010/ 08/better-know-the-klout-classes.

Björneborn, L.; Ingwersen, P. (2004). Toward a basic framework for webometrics. // Journal of the American Society for Information Science and Technology. 55:14, 1216-1227.

Cha, M.; Benevenuto, F.; Haddadi, H.; Gummadi, K. (2012). The world of connections and information flow in Twitter. Systems, Man and Cybernetics, Part A: Systems and Humans. // IEEE Transactions on. 42:4, 991-998.

Chen, S.-C.; Yen, D. C.; Hwang, M. I. (2012). Factors influencing the continuance intention to the usage of Web 2.0: An empirical study. // Computers in Human Behavior. 28:3, 933-941.

Edwards, C.; Spence, P. R.; Gentile, C. J.; Edwards, A.; Edwards, A. (2013). How much Klout do you have... A test of system generated cues on source credibility. // Computers in Human Behavior. 29:5, A12-A16.

Garcia Esparza, S.; O'Mahony, M. P.; Smyth, B. (2012). Mining the realtime web: a novel approach to product recommendation. // Knowledge-Based Systems. 29, 3-11.

Heimeriks, G.; Van den Besselaar, P. (2006). Analyzing hyperlinks networks: The meaning of hyperlink based indicators of knowledge production // Cybermetrics. 10:1.

Holmberg, K. (2010). Co-inlinking to a municipal Web space: a webometric and content analysis. // Scientometrics. 83:3, 851-862.

Holmberg, K.; Thelwall, M. (2009). Local government web sites in Finland: A geographic and webometric analysis. // Scientometrics. 79:1, 157-169.

Ingwersen, P. (1998). The calculation of web impact factors. // Journal of documentation. 54:2, 236-243.

Java, A.; Song, X.; Finin, T.; Tseng, B. (2009). Why we twitter: An analysis of a microblogging community $\mathrm{Ad}-$ vances in Web Mining and Web Usage Analysis. Berlin: Springer. 118-138.

Lee, K.; Palsetia, D.; Narayanan, R.; Patwary, M. M. A.; Agrawal, A.; Choudhary, A. (2011). Twitter trending topic classification. Paper presented at the Data Mining Workshops (ICDMW), 2011 IEEE 11th International Conference.
Metzger, M. J.; Flanagin, A. J.; Medders, R. B. (2010). Social and heuristic approaches to credibility evaluation online. // Journal of Communication. 60:3, 413-439.

Narr, S.; De Luca, E. W.; Albayrak, S. (2011). Extracting semantic annotations from twitter. Paper presented at the Proceedings of the fourth workshop on Exploiting semantic annotations in information retrieval, New Yotk, 15-16

Ortega Priego, J. (2003). A Vector Space Model as a methodological approach to the Triple Helix dimensionality: A comparative study of Biology and Biomedicine Centres of two European National Research Councils from a Webometric view. // Scientometrics. 58:2, 429-443.

Ortega Priego, J.; Aguillo Caño, I. (2007a). Interdisciplinary relationships in the Spanish academic web space: $A$ Webometric study through networks visua-lization. // Cybermetrics. 11:1

Ortega, J.; Aguillo, I. (2007b). La web académica española en el contexto del Espacio Europeo de Educación Superior: estudio exploratorio. // El profesional de la información. 16, 417-425.

Ortega, J.; Aguillo, I. (2008a). Linking patterns in European Union countries: geographical maps of the European academic web space. // Journal of Information Science. 34:5, 705-714.

Ortega, J.; Aguillo, I. (2008b). Visualization of the Nordic academic web: Link analysis using social network tools. // Information Processing \& Management. 44:4, 16241633.

Ortega, J.; Aguillo, I. F. (2008c). Germany in the European academic web space. // Proceedings of WIS 2008, URL: http://www.researchgate.net/publication/228411431_Ger many_in the European_academic_web_space/file/9 $\mathrm{fcfd}$ 50a34e 50 d1392.pdf.

Ortega, J.; Aguillo, I.; Cothey, V.; Scharnhorst, A. (2008d). Maps of the academic web in the European Higher Education Area-an exploration of visual web indicators. // Scientometrics. 74, 295-308

Payne, N.; Thelwall, M. A (2004). Statistical Analysis of UK Academic Web Links Cybermetrics. 8:1.

Serrano-Puche, J. (2012). Herramientas web para la medición de la influencia digital: análisis de klout y Peerlndex. // El profesional de la información. 21:3, 298-303.

Stuart, D.; Thelwall, M.; Harries, G. (2007). UK academic web links and collaboration-an exploratory study. /I Journal of Information Science. 33:2, 231-246

Thelwall, M. (2001). A Web Crawler Design for Data Mining. // Journal of Information Science. 27:5, 319-325.

Thelwall, M. (2002). Conceptualizing documentation on the Web: an evaluation of different heuristic-based models for counting links between university web sites. // Journal of the American Society for Information Science and Technology. 53:12, 995-1005.

Thelwall, M. (2003). What is this link doing here? Beginning a fine-grained process of identifying reasons for academic hyperlink creation. // Information research. 8:3, 3-8.

Thelwall, M.; Harries, G. (2003). The connection between the research of a university and counts of links to its Web pages: An investigation based upon a classification of the relationships of pages to the research of the host university. // Journal of the American Society for Information Science and Technology. 54:7, 594-602

Thelwall, M.; Tang, R.; Price, L. (2003). Linguistic patterns of academic Web use in Western Europe. // Scientometrics. 56, 417-432

Thelwall, M.; Zuccala, A. (2008). A university-centred European Union link analysis. // Scientometrics. 75, 407-420. 
Thelwall, M.; Buckley, K.; Paltoglou, G. (2011). Sentiment in Twitter events. // Journal of the American Society for Information Science and Technology. 62:2, 406-418.

Westerman, D.; Spence, P. R.; Van Der Heide, B. (2012). A social network as information: The effect of system generated reports of connectedness on credibility on Twitter. // Computers in Human Behavior. 28:1, 199-206.

Wilkinson, D.; Harries, G.; Thelwall, M.; Price, L. (2003). Motivations for academic Web site interlinking: evidence for the Web as a novel source of information on informal scholarly communication. // Journal of Information Science. 29:1, 49-56.

Yerva, S. R.; Miklós, Z.; Aberer, K. (2012). Quality-aware similarity assessment for entity matching in Web data. // Information Systems. 37:4, 336-351.

Enviado: 2013-04-05.

Aceptado: 2013-06-23.

Figuerola, Carlos G.; Alonso Berrocal, José Luis; Zazo, Ángel F. Estructuras de colaboración entre las bibliotecas nacionales de la Comunidad Europea a través de sus portales web y de las redes sociales. // Scire. 19:2 (jul.-dic. 2013) 33-44. ISSN 1135-3716. 